\title{
Curve classes on irreducible holomorphic symplectic varieties
}

\author{
Giovanni Mongardi and John Christian Ottem
}

\begin{abstract}
We prove that the integral Hodge conjecture holds for 1-cycles on irreducible holomorphic symplectic varieties of $K 3^{[n]}$-type and of generalized Kummer type. As an application, we give a new proof of the integral Hodge conjecture for cubic fourfolds.
\end{abstract}

Let $X$ be a smooth complex projective variety of dimension $n$. We write $H^{p, p}(X, \mathbb{Z})=$ $H^{2 p}(X, \mathbb{Z}) \cap H^{p, p}(X, \mathbb{C})$ for the group of integral degree $2 p$ Hodge classes, and say that the integral Hodge Conjecture holds for $k$-cycles on $X$ if $H^{n-k, n-k}(X, \mathbb{Z})$ is generated by classes of $k$-dimensional algebraic subvarieties on $X$. While the usual Hodge conjecture predicts that this statement should be true with $\mathbb{Q}$-coefficients, it is known that the integral Hodge conjecture can fail in general, even for 1-cycles [BO, BCC, Tot13, Voi07]. For 1-cycles, the validity of the conjecture depends very much on the birational properties of $X$ (in particular the group of degree $2 n-2$ Hodge classes modulo algebraic classes is a birational invariant). For instance, Voisin showed that the conjecture holds for threefolds that are either uniruled; or satisfy $K_{X}=0$ and $H^{2}\left(X, \mathcal{O}_{X}\right)=0$ [Voi]. For varieties with $K_{X}=0$ of higher dimension (in particular, abelian fourfolds), not much is known.

In this paper we prove that the integral Hodge conjecture holds for 1-cycles on certain irreducible holomorphic symplectic varieties. We consider varieties of $K 3^{[n]}$-type (deformation equivalent to Hilbert schemes of $n$ points on K3 surfaces) and generalized Kummer type (see Section 1). Our main theorem is the following:

Theorem 0.1. Let $X$ be a projective holomorphic symplectic variety of $K 3^{[n]}$-type or of generalized Kummer type. Then the integral Hodge conjecture holds for 1-cycles on $X$.

In fact, the group $H^{2 n-1,2 n-1}(X, \mathbb{Z})$ is generated by classes of rational curves. This can be extended as follows:

Theorem 0.2. Let $X$ be a projective holomorphic symplectic variety of $K 3^{[n]}$-type or of generalized Kummer type. Then the semigroup of effective curve classes is generated (over $\mathbb{Z}$ ) by classes of rational curves.

The above theorems apply in particular to the variety of lines on a cubic fourfold. In this setting, Shen [Sh] proved that the integral Hodge conjecture is related to the algebraicity of the Beauville-Bogomolov form (see Section 5). Using the incidence correspondence, we also give a new proof of the following result of Voisin: 
Corollary 0.3. The integral Hodge conjecture holds for 2-cycles on cubic fourfolds.

The proofs in this paper rely on several results and constructions that were already in the literature. In particular, Theorems 0.1 and 0.2 involve a deformation argument similar to that in $[\mathrm{AV}]$ and $[\mathrm{CMP}]$. We first consider the Hilbert scheme of a K3 or a generalized Kummer variety, where we exhibit special families of rational curves that represent primitive classes in $H^{2 n-2}(X, \mathbb{Z})$, and which also deform in their Hodge loci. This then in turn implies that any integral degree $2 n-2$ Hodge class on a deformation is represented by a rational curve.

We would like to thank O. Benoist, G. Pacienza, M. Shen and C. Vial for useful discussions. GM was supported by the project "2013/10/E/ST1/00688" and "National Group for Algebraic and Geometric Structures, and their Application" (GNSAGA INdAM), and JCO was supported by an RCN grant no. 250104.

\section{Preliminaries}

We work over the complex numbers. An irreducible holomorphic symplectic variety (IHS) $X$ is a simply connected Calabi-Yau manifold carrying a non-degenerate holomorphic 2-form $\omega$ generating $H^{2,0}(X)$. Such a variety $X$ admits a Beauville-Bogomolov form $q$, which is a non-degenerate quadratic form on $H^{2}(X, \mathbb{Z})$.

\subsection{Varieties of $K 3^{[n]}$-type}

Let $S$ be a complex K3 surface and let $S^{[n]}$ denote its Hilbert scheme (or Douady space in the non-projective setting) of length $n$ subschemes of $S$. This is a smooth IHS variety of dimension $2 n$. In general, we say that an IHS variety is of $K 3^{[n]}$-type if it is deformation equivalent to $S^{[n]}$. For these varieties, the Beauville-Bogomolov form $q$ has signature $(3,20)$.

The (co)homology groups of $X=S^{[n]}$ of degree 2 and $4 n-2$ are easy to describe. We have the Hilbert-Chow morphism $H C: S^{[n]} \rightarrow S^{(n)}$ which induces an injection $H^{2}\left(S^{(n)}, \mathbb{Z}\right) \rightarrow H^{2}(X, \mathbb{Z})$ and an injective map $i: H^{2}(S, \mathbb{Z}) \rightarrow H^{2}(X, \mathbb{Z})$ obtained by symmetrizing a line bundle on $S$. From these we obtain decompositions

$$
H^{2}(X, \mathbb{Z})=H^{2}(S, \mathbb{Z}) \oplus \mathbb{Z} B
$$

and

$$
H_{2}(X, \mathbb{Z})=H_{2}(S, \mathbb{Z}) \oplus \mathbb{Z} \tau
$$

where $B=\frac{1}{2}[E]$ is one half of the class of the exceptional divisor $E$ of $H C$ and $\tau$ is the class of a rational curve in a fiber of $\left.H C\right|_{E}$. In particular, we see that the integral Hodge conjecture holds automatically for 1-cycles on $X$, since it holds on $S$.

Viewing $H_{2}(X, \mathbb{Z})$ as $\operatorname{Hom}\left(H^{2}(X, \mathbb{Z}), \mathbb{Z}\right)$, we see that $q$ defines an embedding of lattices

$$
\phi: H^{2}(X, \mathbb{Z}) \rightarrow H_{2}(X, \mathbb{Z})=H^{4 n-2}(X, \mathbb{Z}) .
$$

Over $\mathbb{Q}$ this defines an isomorphism $\phi: H^{2}(X, \mathbb{Q}) \rightarrow H_{2}(X, \mathbb{Q})$, and we extend $q$ to a $\mathbb{Q}$-valued quadratic form on $H_{2}(X, \mathbb{Q})$ and $H_{2}(X, \mathbb{Z})$. In this setting both of the decompositions above are orthogonal with respect to the form $q$. 
Lemma 1.1. Let $X$ be an IHS manifold of dimension $2 n$ and let $D \in H^{2}(X, \mathbb{Z})$ with $q(D) \neq 0$. Let $D^{2 n-1} \in H^{4 n-2}(X, \mathbb{Z})$. Then the classes $\phi(D)$ and $D^{2 n-1}$ are proportional (where $\phi$ is as in (3)). In particular, their Hodge loci inside $\operatorname{Def}(X)$ coincide.

Proof. For a divisor $D$, Fujiki's relation states that $D^{2 n}=c q(D)^{n}$ for a rational constant $c>0$. If $q(D) \neq 0$, this implies that the two integral degree $4 n-2$ classes $D^{2 n-1}$ and $\phi([D])$ are proportional in $H^{4 n-2}(X, \mathbb{Z})$. Therefore, if one stays of Hodge type so does the other, and our claim follows.

\subsection{Generalized Kummer varieties}

Let now $S$ denote an abelian surface and let $n$ be a positive integer. We define the generalized Kummer variety $X=K_{n}(S) \subset S^{[n+1]}$ associated to $S$ as the fiber over 0 of the summation map $S^{[n+1]} \rightarrow S$. Smooth deformations of these varieties are said to be of generalized Kummer type.

As in the previous case, we have a decomposition

$$
H^{2}(X, \mathbb{Z})=H^{2}(S, \mathbb{Z}) \oplus \mathbb{Z} e
$$

where $e=\frac{1}{2} E$ where $E$ is the restriction of the Hilbert-Chow divisor on $S^{[n+1]}$, and

$$
H_{2}(X, \mathbb{Z})=H_{2}(S, \mathbb{Z}) \oplus \mathbb{Z} \eta
$$

where $\eta$ is the class of a minimal curve in the fibres of $H C_{\mid E}$ (thus $\eta$ is represented by a rational curve). Both of these are orthogonal with respect to the Beauville-Bogomolov form.

\subsection{Deforming rational curves}

Let $X$ be an IHS variety of dimension $2 n$ and let $f: \mathbb{P}^{1} \rightarrow X$ be a non-constant map. Let $R$ denote the image of $f$ and let $\operatorname{Def}(X,[R])$ be the sublocus of the local deformation space $\operatorname{Def}(X)$ where the class $[R]$ is of Hodge type $(n-1, n-1)$. The deformation theory of the curve $R \subset X$ and the map $f$ is well-understood, by results of Ran [Ran] and later, in this particular case, by Amerik and Verbitsky [AV] and by Charles, Mongardi and Pacienza [CMP]. We can formulate the result we need using the Kontsevich moduli space $\overline{\mathcal{M}}_{0}(X, \beta)$ parameterizing stable maps $f: \mathbb{P}^{1} \rightarrow X$ with image of class $f_{*}\left[\mathbb{P}^{1}\right]=\beta$. If $f: \mathbb{P}^{1} \rightarrow X$ is a finite map, then every component $\mathcal{M} \subseteq \overline{\mathcal{M}}_{0}(X,[R])$ containing the corresponding point $[f]$ has dimension at least $2 n-2$ (cf. [Ran, Corollary 5.1]). Ran's results can then be summarized as follows:

Proposition 1.2. [Ran, Corollaries 3.2, 3.3 and 5.1] Let $X, R, f$ be as above. Suppose there is a component of $\overline{\mathcal{M}}_{0}\left(\mathbb{P}^{1},[R]\right)$ of dimension $2 n-2$ containing $[f]$. Then the curve $R$ deforms in the Hodge locus $\operatorname{Def}(X,[R])$.

In other words, given a family $\pi: \mathcal{X} \rightarrow T$ of IHS varieties with a special fiber $X=\mathcal{X}_{0}$; a rational curve $f: \mathbb{P}^{1} \rightarrow X$ with image $R \subset X$; and a global section of $R^{4 n-2} \pi_{*} \mathbb{Z}$ of Hodge type $(2 n-1,2 n-1)$, specializing to $[R]$ on $X$. Then if there is a component of 
the moduli space $\overline{\mathcal{M}}_{0}(X,[R])$ containing $[f]$ of dimension exactly $2 n-2$, then the map $f: \mathbb{P}^{1} \rightarrow \mathcal{X}$ deforms (after taking some finite cover of $T$ ), and in particular $R$ deforms in the fibers of $\pi$. (See also [CMP, Section 3] and [AV] for similar statements).

\section{Special rational curves}

To prove Theorems 0.1 and 0.2 , we will need to construct certain special rational curves on $S^{[n]}$ and $K_{n}(S)$ that satisfy the conditions of Proposition 1.2. To explain the basic idea, consider the case where $n=2$, and $S$ is a K3 surface of degree 2. Letting $H$ be the polarization on $S$, each smooth curve $C \in|H|$ is a genus 2 curve admitting a unique $\mathfrak{g}_{2}^{1}$. This defines a rational curve $R_{C}$ on $C^{[2]}$, and hence on $S^{[2]}$. We can write the class $\left[R_{C}\right] \in H_{2}\left(S^{[2]}, \mathbb{Z}\right)$ in terms of the decomposition (2); intersecting $R_{C}$ with $H$ shows that it has the form $H-t B$ for some $t \in \mathbb{Z}$. In particular, the class is primitive. From the double cover $S \rightarrow \mathbb{P}^{2}$, we also obtain a plane $\mathbb{P}^{2} \subset S^{[2]}$, which contains all the curves $R_{C}$, and the plane can be contracted by a birational map (see [OG, Section 1.3] for more details). In particular $R_{C}$ deforms in a family of dimension 2 and thus satisfies Proposition 1.2.

The example above is special in the sense that the curves $R_{C}$ are smooth. To construct other classes on $S^{[n]}$, we need to consider singular curves on $S$ and linear series on their normalizations. For this, we apply results on Brill-Noether theory on nodal curves on surfaces due to Ciliberto and Knutsen [CK] for $K 3$ surfaces, and by Knutsen, Lelli-Chiesa and the first author [KLM] for abelian surfaces.

Let us start with Hilbert schemes of $\mathrm{K} 3$ surfaces. Let $(S, H)$ be a primitively polarized K3 surface of degree $H^{2}=2 p-2$ and let $C \in|H|$ be a curve with $\delta$ nodes as its only singular points. Given a linear series $\mathfrak{g}_{n}^{1}$ on the normalization $\widetilde{C}$ of $C$, we obtain a natural rational curve $R_{C}$ in $S^{[n]}$ via the incidence correspondence

$$
I=\left\{(P,[Z]) \in S \times S^{[n]} \mid P \in \operatorname{Supp}(Z)\right\} \rightarrow S^{[n]} .
$$

We say that the nodes are 'non-neutral' with respect to the linear series if the linear series is base point free, and the corresponding morphism $\widetilde{C} \rightarrow \mathbb{P}^{1}$ has simple ramification and does not ramify over the nodes of $C$. If this genericity condition is satisfied, we have that the homology class of $R_{C}$ is given in terms of the decomposition (2) as

$$
H-(p-\delta+n-1) \tau,
$$

where $H \in \operatorname{Pic}(S)$ (see [KLM2][Lemma 3.3]). Note that this class is primitive, since $H$ is. Moreover, the following divisor is proportial to $R_{C}$ via (3):

$$
(2 n-2) H-(p-\delta+n-1) B .
$$

The main properties of these curves are summarized in the following theorem. In the theorem, $V_{|H|, \delta}^{n} \subseteq|H|$ denotes the Severi variety of curves $C$ with $\delta$ nodes, whose normalizations admit a $\mathfrak{g}_{n}^{1}$.

Theorem 2.1. [CK, Thm 0.1] Let $(S, H)$ be a very general primitively polarized $K 3$ of genus $p:=p_{a}(H) \geq 2$. Let $\delta$ and $n$ be integers satisfying $0 \leq \delta \leq p$ and $n \geq 2$. Then the following statements hold: 
(i) $V_{|H|, \delta}^{n}$ is non-empty if and only if

$$
\delta \geq \alpha(p-\delta-(n-1)(\alpha+1)), \text { where } \alpha=\left\lfloor\frac{p-\delta}{2 n-2}\right\rfloor
$$

(ii) Whenever non-empty, $V_{|H|, \delta}^{n}$ is equidimensional of the expected dimension $\min \{2 n-$ $2, p-\delta\}$, and a general point on each component corresponds to an irreducible curve with normalization $\widetilde{C}$ of genus $g=p-\delta$, such that the set of $\mathfrak{g}_{n}^{1}$ 's on $\widetilde{C}$ is of dimension $\max \{0,2 n-2-g\}$.

(iii) There is a component $V \subseteq V_{|H|, \delta}^{n}$, so that for $C$ and $\widetilde{C}$ as in $(i i)$, a general $\mathfrak{g}_{n}^{1}$ on $\widetilde{C}$ is base point free and all nodes of $C$ are non-neutral with respect to it.

So if $p-\delta \geq 2 n-2, V_{|H|, \delta}^{n}$ has dimension $2 n-2$ and the set of $\mathfrak{g}_{n}^{1}$ on a general curve is 0-dimensional. When $p-\delta \leq 2 n-2, V_{|H|, \delta}^{n}$ has dimension $p-\delta$ and the set of rational curves $R_{C}$ on $S^{[2]}$ has dimension $(p-\delta)+(2 n-2-g)=2 n-2$. Hence in either case we have, if the above inequality is satisfied, a family of rational curves on $S^{[n]}$ of dimension $2 n-2$.

There is a very similar statement in the generalized Kummer case. We consider a primitively polarized abelian surface $(S, H)$ of degree $H^{2}=2 p-2$. Let $C$ be an element of $\{H\}$, the continuous system of curves with cohomology class $H$ (that is, $|H|$ up to translations on $S$ ). As before, given a linear series $\mathfrak{g}_{n+1}^{1}$ on the normalization of $C$, we obtain a natural rational curve $R_{C}$ in $K_{n}(S)$. If the nodes are non-neutral with respect to this series, the class of $R_{C}$ is given by

$$
H-(p-\delta+n) \eta
$$

This is again primitive, if $H$ is.

Theorem 2.2. [KLM, Theorem 1.6] Let $(S, H)$ be a very general abelian surface of genus $p:=p_{a}(H) \geq 2$. Let $\delta$ and $n$ be integers satisfying $0 \leq \delta \leq p-2$ and $n \geq 2$. Then the following hold:

(i) There exists a nodal curve in $\{H\}$ whose normalization has a linear series of type $\mathfrak{g}_{n+1}^{1}$ if and only if

$$
\delta \geq \alpha(p-\delta-1-(n+1)(\alpha+1)), \text { where } \alpha=\left\lfloor\frac{p-\delta-1}{2 n+2}\right\rfloor ;
$$

(ii) When non-empty, the set of $\mathfrak{g}_{n+1}^{1}$ 's on curves in $\{H\}$ with $\delta$ nodes is equidimensional of dimension $\min \{p-\delta, 2 n\}$ and a general element in each component is an irreducible curve $C$ with normalization $\widetilde{C}$ of genus $g:=p-\delta$ such that it has $a \max \{0, \rho(g, 1, n+1)\}=2 n-g$ dimensional set of $\mathfrak{g}_{n+1}^{1}$ 's;

(iii) There is at least one component of the above locus where, for $C$ and $\widetilde{C}$ as in (ii), a general $\mathfrak{g}_{n+1}^{1}$ on $\widetilde{C}$ is base point free and all nodes of $C$ are non-neutral with respect to it. 
Let us illustrate how to use this theorem to construct rational curves in a few interesting cases on Hilbert schemes of $n$ points on K3 surfaces.

Example 2.3. Consider a polarized K3 surface $(S, H)$ with $H^{2}=2 d$, and the curve class $\gamma=H-(n-1) B$ on $X=S^{[n]}$. Then $\gamma$ would be represented by a rational curve $R \subset X$ corresponding to a linear series of type $\mathfrak{g}_{n}^{1}$ on the normalization of a rational curve in $|H|$. It is clear that such linear series exist on $\mathbb{P}^{1}$, so for any $n>0$, we obtain rational curves on $S^{[n]}$, which move in a $(2 n-2)$-dimensional family.

In this example, the dual divisor of $\gamma$ is given by $D=2 H-B$. We will see below (Lemma 2.9) that it is possible to deform any pair $\left(X, D^{\prime}\right)$ of $K 3^{[n]}$-type $X$ with a divisor $D^{\prime}$ of square $8 d-(2 n-2)$ and divisibility 2 to a pair $\left(S^{[n]}, D\right){ }^{1}$

Example 2.4. A similar situation arises for the class $H-(2 n-1) \tau$ on $S^{[n]}$. This corresponds to linear series of type $\mathfrak{g}_{n}^{1}$ on normalizations of curves on $S$ of geometric genus $n$ inside $|H|$. If $q(H-(2 n-1) \tau)>0$, the square of $H$ is strictly bigger than $2 n-2$, so there does indeed exist curves of genus $n$ inside $|H|$ (here the integer $\alpha$ in (6) is zero, and the conditions of Theorem 2.1 are satisfied). Since any smooth curve of genus $n$ contains a $\mathfrak{g}_{n}^{1}$, we obtain the desired rational curves on $S^{[n]}$.

Example 2.5. Let $F$ denote the Fano variety of lines of a cubic fourfold $Y \subset \mathbb{P}^{5}$. It is well-known that $F$ is deformation equivalent to a Hilbert scheme $S^{[2]}$ of a K3 surface $(S, H)$ with $H^{2}=14$. In terms of $H$ and $B$, the Plücker polarization $\mathcal{O}_{F}(1)$ from the ambient Grassmannian $\operatorname{Gr}(2,6)$ is given by $\mathcal{O}(1)=2 H-5 B$ (see [BD]). The dual curve class is given by $H-5 \tau$. We can produce rational curves on $S^{[2]}$ with this class using Theorem 2.1 by taking $p=8, \delta=4$. Then $\alpha=2$ and the inequality (6) is satisfied. In this example, we obtain a 2-dimensional family of rational curves, since $V_{|H|, \delta}^{n}$ has dimension 2 , and each normalization $\widetilde{C}$ has at most one $\mathfrak{g}_{2}^{1}$.

These rational curves have degree $(2 H-5 B) \cdot(H-5 \tau)=2 \cdot 14-25=3$. Geometrically the curves arise as follows: For each line $\ell$ of 'type II' (that is, the normal bundle splits as $\left.\mathcal{O}(1)^{2} \oplus \mathcal{O}(-1)\right)$, there is a tangent $\mathbb{P}^{3}$ which intersects $Y$ in a cubic surface singular along $\ell$; the residual lines are parameterized by a degree 3 curve in $F$.

More generally, we have the following result for K3 surfaces, with a straightforward generalization to the generalized Kummer case (with $2 n+3 \leq \mu \leq 3 n+3$ ):

Lemma 2.6. Let $S$ be a K3 surface of degree $2 d$ and let $2 n-1 \leq \mu \leq 3 n-3$ be an integer. Let $R$ be the curve class $H-\mu \tau$ in $S^{[n]}$. Suppose $q(R) \geq 0$. Then there exists a rational curve of class $R$ and a component $\mathcal{M} \subseteq \overline{\mathcal{M}}_{0}(X,[R])$ of dimension $2 n-2$.

Proof. We want to construct $R$ as in the previous examples. That is, we are trying to construct linear series of type $\mathfrak{g}_{n}^{1}$ on curves in $|H|$ of geometric genus $\mu-n+1$. By the bound on $\mu$, we have $\alpha=0$ unless $n=2$ or $\mu=3 n-3$, where $\alpha$ is as in Theorem 2.1. Therefore, if we are not in these cases, the bound of Theorem 2.1 is satisfied and the linear series exists if and only if there are curves in $|H|$ of genus $\mu-(n-1)$. Since $H$

\footnotetext{
${ }^{1}$ Divisibility 2 means that $\left\{q\left(D^{\prime}, x\right) \mid x \in H^{2}(X, \mathbb{Z})\right\}=2 \mathbb{Z}$. An easy computation shows that the square of any divisor of divisibility 2 is congruent to $(2-2 n)$ modulo 8 , so the above statement in fact applies to any divisor of divisibility 2 .
} 
has genus $d+1$, this happens if and only if $d+1 \geq \mu-(n-1)$. However, by assumption, $q(R)=2 d-\frac{\mu^{2}}{2 n-2}$ is positive, and this implies $d+1 \geq \mu-(n-1)$. The remaining cases when $n=2$ or $\mu=3 n-3$ have $\alpha=1$, but the condition of Theorem 2.1 is trivially satisfied, so the above applies also to this case. By item (ii) of Theorem 2.1, the curve $R$ deforms in a family of dimension $2 n-2$. Moreover, the incidence correspondence (4) can be used to prove that all deformations of these rational curves on the Hilbert scheme of points on a general K3 (or a generalized Kummer) are actually induced by linear series of type $\mathfrak{g}_{n}^{1}$ on curves in the closure of $V_{|H|, d-\mu+n}^{n}$ (see [KLM, Proposition 5.3] or [KLM2, Proposition 3.6] for a proof of this).

In particular, this implies that all curves constructed using the above theorems satisfy the hypothesis of Proposition 1.2. This fact will be important in the proof of Theorem 0.1 and Lemma 3.2 below. Moreover, we will construct similar curves to prove Lemma 4.2 and then finally Theorem 0.2 .

Remark 2.7. These proofs of the two theorems above rely on the assumption that the Picard number of $S$ is 1 . If $S$ has higher Picard number, these curves can move in larger families, as was exploited in [FKP] to construct rational surfaces.

Finally, let us conclude with a lattice theoretic result which highlights the importance of Lemma 2.6. An analogous result was proven in [CMP, Theorem 2.5 and Corollary 2.8], with different values of $\mu$. We include a proof for completeness. Let $L$ be an even lattice and let $A_{L}:=L^{\vee} / L$ be its discriminant group, which is a finite group. For the properties of the discriminant group and its links to lattice theory, we refer to [N]. Given a primitive element $l \in L$, we define the divisibility $\operatorname{div}(l)$ as the positive generator of the ideal $(l, L)$ in $\mathbb{Z}$. The element $l / \operatorname{div}(l)$ is then a well defined element of $L^{\vee}$ and we denote by $[l / \operatorname{div}(l)]$ its class in $A_{L}$. We will use the following, known as Eichler's criterion:

Lemma 2.8. [GHS, Lemma 3.5] Let $L^{\prime}$ be an even lattice and let $L=U^{2} \oplus L^{\prime}$. Let $v, w \in L$ be two primitive elements such that the following holds:

- $v^{2}=w^{2}$.

- $[v / \operatorname{div}(v)]=[w / \operatorname{div}(w)]$ in $A_{L}$.

Then there exists an isometry $g \in \widetilde{O}^{+}(L)$ with determinant one and such that $g(v)=w$.

Here, $U$ denotes the rank 2 hyperbolic lattice, $O^{+}(L)$ denotes the group of isometries preserving the orientation on $L$ and $\widetilde{O}^{+}(L)$ denotes the subgroup of isometries whose induced action on $A_{L}$ is trivial. Note that, if $L$ is primitively embedded into another lattice $M$, an isometry of $\widetilde{O}(L)$ can be extended to an isometry of $M$ acting trivially on $L^{\perp}$.

Lemma 2.9. Let $(X, D)$ be a pair consisting of a manifold $X$ of $K 3^{[n]}$-type and a divisor $D$ of square $2 d$ and divisibility $t$. Then there exists a polarized $K 3$ surface $(S, H)$ of degree $2 s$, an integer $2 n-1 \leq \mu \leq 3 n-3$ such that $(X, D)$ is deformation equivalent ${ }^{2}$ to $\left(S^{[n]}, t H-\mu / e B\right)$, where $e=\operatorname{gcd}(2 n-2, \mu)$ and the dual curve to $t H-\mu / e B$ is $H-\mu \tau$.

\footnotetext{
${ }^{2}$ See Section 3 for the notion of deformation equivalence.
} 
Proof. First of all, the pair $(X, D)$ can be deformed to a pair $\left(S^{[n]}, D^{\prime}\right)$ for some $S$ and some $D^{\prime}$, as the locus of Hilbert schemes is an hyperplane divisor in the marked moduli space of manifolds of $K 3^{[n]}$-type, hence it intersects the image of the moduli space of pairs $(X, D)$ in the period domain, which is path connected, and by [Ma, Proposition 7.1] this path lifts to a deformation of $(X, D)$ to $\left(S^{[n]}, D^{\prime}\right)$. We can further deform $S$ so that it is very general and has Picard rank one, with generator $H$, therefore $D^{\prime}=a H+b B$, where $t$ divides $a$ and $D^{\prime 2}=D^{2}$. By [Ma, Corollary 9.5], there is an embedding of $\Lambda_{n}:=H^{2}\left(S^{[n]}, \mathbb{Z}\right)$ in the Mukai lattice $\Lambda:=U^{4} \oplus E_{8}(-1)^{2}$, which is well defined up to isometry and coincides with the usual embedding obtained by seeing $S^{[n]}$ as a moduli space of ideal sheaves on $S$. Notice that, if $n-1$ is a prime power, there is only one isometry class of these embeddings. Moreover, the orthogonal complement of $\Lambda_{n}$ under this embedding has rank one, and let $v$ be a generator of it.

Let us denote by $T\left(D^{\prime}\right)$ and $T(t H-\mu / e B)$ the rank two primitive lattices containing $D^{\prime}$ and $v$ or $t H-\mu / e B$ and $v$ respectively. By [Ap, Proposition 1.6], the two pairs are deformation equivalent if and only if there exists an isometry of $T\left(D^{\prime}\right)$ and $T(t H-\mu / e B)$ sending $D^{\prime}$ to $t H-\mu / e B$. That is, the isometry must send $v$ into $\pm v$ and therefore descends to an isometry of $\Lambda_{n}$. Let us consider the discriminant group $A_{\Lambda_{n}}:=\Lambda_{n}^{\vee} / \Lambda_{n}$, which is a cyclic group of order $2 n-2$ generated by $[B /(2 n-2)]$. By elementary lattice theory, the isometry above has to act as \pm 1 on the discriminant group $A_{\Lambda_{n}}$, see e.g. [Ma, Corollary 9.5]. Notice that $D^{\prime} / t$ and $(t H-\mu / e B) / t=(H-\mu /(2 n-2) B)$ are well defined elements of $\Lambda_{n}^{\vee}$ by the definition of divisibility.

Notice that $[(t H-\mu / e B) / t]$ is equal to $\mu[B /(2 n-2)]$ inside $A_{\Lambda_{n}}$. From Eichler's criterion in Lemma 2.8, if $\left[D^{\prime} / t\right]=[(t H-\mu / e B) / t]$ there exists an isometry of $\Lambda_{n}$ which acts trivially on $A_{\Lambda_{n}}$ (and therefore can be extended to $\Lambda$ ) sending $D^{\prime}$ to $t H-\mu / e B$. Therefore our claim holds true with $2 n-1 \leq \mu \leq 4 n-4$, so that $\mu$ takes all possible values modulo $2 n-2$. To obtain the desired bound, it suffices to compose with the isometry given by reflection along $B$, which acts as -1 on $A_{\Lambda_{n}}$, so that only half of the values of $\mu$ are needed.

Remark 2.10. In the case of a pair $(X, D)$ consisting of a manifold $X$ of generalized Kummer type and a divisor $D$ of square $2 d$ and divisibility $t$, an analogous argument to [MP, Theorem 4.2] and the above lemma allows to prove that there exists a polarized abelian surface $(S, H)$ of degree $2 s$, an integer $2 n+3 \leq \mu \leq 3 n+3$ such that $(X, D)$ is deformation equivalent to $\left(K_{n}(S), t H-\mu / c e\right)$, where $c=\operatorname{gcd}(2 n-2, \mu)$ and the dual curve to $t H-\mu / c e$ is $H-\mu \eta$.

\section{Proof of Theorem 0.1}

Let $X, X^{\prime}$ be two IHS varieties and let $h, h^{\prime}$ be primitive polarizations on $X$ and $X^{\prime}$ respectively. We will for simplicity say that $(X, h)$ and $\left(X^{\prime}, h^{\prime}\right)$ are deformation equivalent if there is a smooth projective family $\pi: \mathcal{X} \rightarrow T$ over an irreducible curve $T$; a line bundle $\mathscr{H}$ on $\mathcal{X}$; and two points $0,1 \in T$ so that $(X, h)=\left(\mathcal{X}_{0},\left.c_{1}(\mathscr{H})\right|_{\mathcal{X}_{0}}\right)$ and $\left(X^{\prime}, h^{\prime}\right)=\left(\mathcal{X}_{1},\left.c_{1}(\mathscr{H})\right|_{\mathcal{X}_{1}}\right)$. 
Lemma 3.1. Let $(X, h)$ and $\left(X^{\prime}, h^{\prime}\right)$ be two deformation equivalent primitively polarized IHS varieties of dimension $2 n$, connected by a family $\pi: \mathcal{X} \rightarrow T$, and let $R \subset X$ be a rational curve which deforms in a family of dimension $2 n-2$ in $X$. Suppose further that $[R]$ is proportional to $h$ (via the embedding (3)). Then $R$ deforms in the fibres of $\pi$. In particular, also $X^{\prime}$ has an effective 1-cycle (with class proportional to $h^{\prime}$ ) with components being rational curves.

Proof. Let $f: \mathbb{P}^{1} \rightarrow X$ denote the stable map corresponding to $R$, so that $[R]=f_{*}\left[\mathbb{P}^{1}\right]$. Since the deformation space has an irreducible component of dimension $2 n-2=$ $\operatorname{dim} X-2$, we have, by Proposition 1.2, that the curve deforms in its Hodge locus. By assumption, our class $[R]$ is Hodge on all the fibers of $\pi$, since it is proportional to the restriction of the $(1,1)$-class $c_{1}(\mathscr{H})$ on $\mathcal{X}$. Since $T$ is irreducible, this means that $R$ deforms to a chain of rational curves on $X^{\prime}$.

Theorem 3.2. Let $X$ be a IHS variety of $K 3^{[n]}$-type or generalized Kummer type of dimension $2 n$, and let $\gamma \in H^{4 n-2}(X, \mathbb{Z})$ be a primitive integral Hodge class with $q(\gamma)>0$. Then $\gamma$ is cohomologous to a sum of rational curves.

Proof. Let $\gamma$ be such a class and let $D$ the primitive divisor proportional to it (under the embedding of (3)). Since $q(D)>0$, there is a deformation $\pi: \mathcal{X} \rightarrow T$ of $X$ over an irreducible curve $T$; points $0,1 \in T$; and a divisor class $\mathcal{D}$ on $\mathcal{X}$, so that $\left(\mathcal{X}_{0}, \mathcal{D}_{0}\right)=(X, D)$ and $\left(\mathcal{X}_{1}, \mathcal{D}_{1}\right)$ is either the Hilbert scheme of a K3 surface $S$ or a generalized Kummer variety of an abelian surface $S$ and we can suppose such a surface has Picard rank one.

The divisor $\mathcal{D}_{1}$ can be chosen to be of the form $t H-\mu \tau$ for $H \in \operatorname{Pic}(S)$ primitive and integers $t, \mu$ satisfying $2 n-1 \leq \mu \leq 3 n-3$ in the $K 3^{[n]}$ case and $2 n+3 \leq \mu \leq 3 n+3$ in the Kummer case by Lemma 2.9 and Remark 2.10 .

Therefore, we can use the curves constructed in Lemma 2.6 so that all possible monodromy orbits are covered. Let us call this rational curve $R^{\prime}$ on $\mathcal{X}_{1}$ so that $\left[R^{\prime}\right]$ is primitive and proportional to $\mathcal{D}_{1}$ (via the embedding (3) and by Lemma 1.1). By construction, there is a corresponding component of $\overline{\mathcal{M}}_{0}\left(X,\left[R^{\prime}\right]\right)$ has dimension exactly $2 n-2$. Since $\mathcal{D}_{1}$ is the restriction of $[\mathcal{D}]$ on $\mathcal{X}$, the class $[R]$ is Hodge in the fibers of $\pi$, and so Lemma 3.1 shows that the map $f: \mathbb{P}^{1} \rightarrow \mathcal{X}$ deforms in a family dominating $T$. In particular, this means that $R$ deforms to a 1-cycle $R^{\prime}$ on $X$, all of whose components are rational. By construction, the class of $R$ this is a multiple of $\gamma$, and so by primitivity $[R]=\gamma$. This completes the proof of the theorem.

With this, we can prove our main theorem:

Proof of Theorem 0.1. The group of integral degree $4 n-2$ Hodge classes is generated by primitive classes with positive Beauville-Bogomolov square, and these have algebraic representatives by the previous theorem.

The proof of the above theorem can likely be applied to other situations. Indeed, the key ingredient of the proof is a statement similar to a conjecture of Voisin [Voi14b, Conj. 3.1 and Remark 3.2], namely the following: 
Conjecture 1. Let $X$ be a projective IHS variety of dimension $2 n$. Then there is a primitive rational curve on $X$ which moves in a $(2 n-2)$-dimensional family.

This conjecture has been proved for varieties of $K 3^{[n]}$-type and Kummer type in [MP2, Theorem 5.1] following ideas contained in [CMP] and [MP].

Theorem 3.3. Let $X$ be a IHS variety such that Conjecture 1 holds for generic projective deformations of $X$. Then the integral Hodge conjecture holds for 1-cycles on $X$.

Indeed, starting with $X$ and an integral class $\gamma \in H^{2 n-1,2 n-1}(X, \mathbb{Z})$ so that $\gamma$ is proportional to a primitive ample divisor class $H \in \operatorname{Pic}(X)$, we can take a very general projective deformation $\left(X^{\prime}, H^{\prime}\right)$ of $(X, H)$ so that $\operatorname{Pic}\left(X^{\prime}\right)=\mathbb{Z} H^{\prime}$. By the above conjecture, there exists a primitive curve on $X^{\prime}$ proportional to $H^{\prime}$ (via the embedding (3)). Moreover, this curve moves in a family of dimension exactly $2 n-2$. Therefore, by Proposition 1.2 such a curve deforms to a 1-cycle on $(X, H)$ representing $\gamma$.

Remark 3.4. If $C$ is a rational curve which is the ruling of a uniruled divisor $D \subset X$, then $C$ moves in a family of exactly dimension $2 n-2$ as proven in [CMP][Corollary 3.5]. However, some of the primitive curves constructed above cannot cover divisors, as was shown in [OSY, Appendix A.3]. In loc. cit, there are necessary condition to ensure that a primitive rational curve covers a divisor, and some examples where this conditions are not met are provided. Nevertheless, the curves we construct in Lemma 2.6 are sufficient to prove our claim.

Remark 3.5. For a non-projective IHS manifold, it is of course not true that the integral degree $4 n-2$ Hodge classes are generated by classes of curves. This fails already in dimension two; there exists Kähler K3 surfaces with $H^{1,1}(X, \mathbb{Z})=\mathbb{Z} \sigma$ for a class with self-intersection $\sigma^{2}=-4$ (which couldn't possibly be algebraic).

\section{Proof of Theorem 0.2}

To prove Theorem 0.2, we need to analyze curves of non-positive Beauville-Bogomolov square. Let us consider first those of negative square.

Lemma 4.1. Let $Y$ be a smooth non-projective IHS manifold of dimension $2 n$, with $\operatorname{Pic}(X)=\mathbb{Z} D$ and $q(D)<0$. Let $\gamma$ be the class of a curve on $Y$. Then $\gamma$ is a multiple of a rational curve which moves in a $2 n-2$ dimensional family.

Proof. As $\gamma$ is effective and of negative square in a Picard rank one manifold, the divisor class $D$ is a wall divisor (or MBM class as in [AV]) in the sense of [Mo, Definition 1.2]. Being a wall divisor is preserved by deformations in the Hodge locus of $\gamma$ by [Mo, Theorem 1.3], so we can take a projective small deformation $Y^{\prime}$ of $Y$ such that $\gamma$ is contracted by a map $Y^{\prime} \rightarrow X^{\prime}$ by [KLM2, Theorem 2.5]. Now, this implies that there exists a map $\phi: Y \rightarrow X$ to a singular symplectic manifold $X$ which contracts $\gamma$ without taking any deformation (see [BL, Theorem 1.1]).

Let $F$ be a general fibre of the exceptional locus of $\phi$. By [Wi2, Theorem 1.3 (iii)], the normalization of any component of $F$ is a projective space $\mathbb{P}^{e}$. Let $K$ be such a 
component and let $\eta: \mathbb{P}^{e} \rightarrow K$ be the normalization map. Let $l \in H_{2}\left(\mathbb{P}^{e}, \mathbb{Z}\right)$ be the class of a line. By hypothesis, we have $\gamma=a \eta_{*}(l)$ as a class in $H_{2}(Y, \mathbb{Q})$ for some $a \in \mathbb{Q}$. Here $a$ is actually an integer: $\eta^{*}(\gamma) \cdot l=a \eta^{*} \eta_{*}(l) \cdot l=a$. Hence $\gamma$ is an integral multiple of a rational curve.

Finally, if $f: \mathbb{P}^{1} \rightarrow X$ is the map corresponding to $l$, we have by [CMSB, Lemma 9.4] that $N_{f}=\mathcal{O}_{\mathbb{P}^{1}}(-2) \oplus \mathcal{O}_{\mathbb{P}^{1}}(-1)^{e-1} \oplus \mathcal{O}_{\mathbb{P}^{1}}^{2 n-2 e} \oplus \mathcal{O}_{\mathbb{P}^{1}}(1)^{e-1}$. In particular, $f$ deforms in a family of dimension $h^{0}\left(N_{f}\right)-h^{1}\left(N_{f}\right)+1=2 n-2$, as desired. (See also [BHT, Proposition $3])$.

Lemma 4.2. Let $Y$ be a smooth non-projective hyperkähler manifold of $K 3^{[n]}$ or Kummer type, with $\operatorname{Pic}(X)=\mathbb{Z} D$ with $D^{2}=0$. Let $\gamma$ be the class of a curve on $Y$. Then $\gamma$ is a multiple of a rational curve which moves in a $(2 n-2)$-dimensional family.

Proof. We can assume that the divisor $D$ is effective, so that it defines a lagrangian fibration structure on $X$ (see [Ma2, Theorem 1.3] and [Mat, Corollary 1.1]).

We will for simplicity consider only the case when $X$ has $K 3^{[n]}$ type. The rest of the proof goes through almost verbatim for generalized Kummer varieties.

First, we want to deform $Y$ to a general Hilbert scheme $S^{[n]}$ where $S$ is a K3 surface. Let $C=\gamma / r, r>0$ be the primitive cohomology class. It suffices to produce a pair $\left(S^{[n]}, H-g \tau\right)$, where $H-g \tau$ is the class of a rational curve, for every component of the moduli space of pairs $(Y, C)$. In this class of square zero curves (actually, divisors) these components have been determined by Markman [Ma2, Lemma 2.5] and Wieneck [Wi1, Lemma 5.12]. All monodromy orbits contain an element of the form $\left(S^{[n]}, H-b \tau\right)$. In particular, it suffices to take $2(n-1)<b \leq 3(n-1)$. Therefore, we can again use Lemma 2.6 to produce a rational curve of class $H-b \tau$ which moves in a $2 n-2$ dimensional family. By Proposition 1.2, this curve deforms to the desired curve on $Y$.

Proof of Theorem 0.2. Let $X$ be a IHS manifold of $K 3^{[n]}$ or Kummer type and let $C$ be a curve on $X$. If $q(C)>0$, then $C$ is an integral sum of rational curves by Theorem 3.2. If $q(C)=0$, the same holds by Lemma 4.2 after going to a generic deformation as in Lemma 3.1. We are left with the case $q(C)<0$. If $C$ is extremal, we can apply Lemma 4.1 to conclude that $C$ is a multiple of a rational curve with the deformation argument of Lemma 3.1. Otherwise, we can apply the Cone theorem of [HT, Proposition 11], so that $C$ can be written as a rational sum of extremal curves $C=\sum a_{i} R_{i}$, where all $R_{i}$ are primitive rational curves by Lemma 4.1.

We show that the coefficients $a_{i}$ are in fact integers. Fix an integer $i$ and let us take a divisor $D_{i} \in \operatorname{Pic}(X)$ which is effective and such that $D_{i} \cdot R_{i}>0, D_{i} \cdot R_{j}<0$ if $i \neq j$. Such a divisor can be found inside the big cone intersected with the open subsets $\left\{D \in N^{1}(X) \mid D \cdot R_{j}<0\right\}$ and $\left\{D \in N^{1}(X) \mid D \cdot R_{i}>0\right\}$.

As a small enough multiple of $D_{i}$ is klt by [HT, Remark 12], we can run the MMP to the pair $\left(X, D_{i}\right)$ contracting negative curves in any order, as every MMP terminates by [LP, Theorem 4.1]. Thus, there exists a variety $Y$ and a map $X \rightarrow Y$ which contracts all curves whose classes are multiples of the $R_{j} j \neq i$. Therefore, the class of the pushforward of $C$ to $Y$ is $a_{i} R_{i}$, where we keep denoting by $R_{i}$ its pushforward to $Y$. As $R_{i}$ is primitive, $a_{i} \in \mathbb{Z}$, and hence the desired conclusion holds. 


\section{Application to cubic fourfolds}

As an application of Theorem 0.1, we give a quick proof of the following result, which was proved by Voisin [Voi07], using an argument using Lefschetz pencils.

Theorem 5.1. Let $X \subset \mathbb{P}^{5}$ be a cubic fourfold. Then the integral Hodge conjecture holds for $H^{4}(X, \mathbb{Z})$. In fact, $H^{2,2}(X, \mathbb{Z})$ is generated by classes of rational surfaces.

Proof. Let $F=F(X)$ denote the variety of lines on $X$ and let $P \subset F \times X$ denote the incidence correspondence, with projections $p: P \rightarrow F$ and $q: P \rightarrow X$. We will consider the (transpose of the) Abel-Jacobi map induced by $P$, namely

$$
\alpha=q_{*} p^{*}: H^{6}(F, \mathbb{Z}) \rightarrow H^{4}(X, \mathbb{Z})
$$

By Beauville-Donagi [BD, Proposition 4], this map is an isomorphism. Hence any integral Hodge class $\Gamma \in H^{2,2}(X, \mathbb{Z})$ is the image of a class in $H^{3,3}(F, \mathbb{Z})$, and consequently it is algebraic, by Theorem 0.1 . The last statement also follows, since the incidence correspondence sends rational curves on $F$ to rational surfaces on $X$.

Remark 5.2. By Bloch-Srinivas [BS, Theorem 1] the cycle class map on codimension 2 cycles is injective, so in fact the Chow group $C H^{2}(X)$ is isomorphic to $H^{2,2}(X, \mathbb{Z})$, and it is generated by cycle classes of rational surfaces. See also the work of Mboro [M] for similar statements for $\mathrm{CH}_{2}(\mathrm{X})$ for higher dimensional cubic hypersurfaces.

Remark 5.3. Using results by Shen [Sh] we obtain the following result about the algebraicity of the Beauville-Bogomolov form. Let $X$ and $F$ be as above. The BeauvilleBogomolov form $q$ on $H^{2}(F, \mathbb{Z})$ defines a class in $H^{12}(F \times F, \mathbb{Z})$ which we will denote by $[q]$. This class is Hodge of type $(6,6)$. Given the validity of the integral Hodge conjecture on $F$, we can rephrase [Sh, Proposition 5.5] as

Proposition 5.4 (Shen). The class $[q]$ is algebraic if and only if $\mathrm{X}$ is $\mathrm{CH}_{0}$-trivial.

Here the group $\mathrm{CH}_{0}(\mathrm{X})$ is universally trivial if and only if $\mathrm{X}$ admits a Chowtheoretical decomposition of the diagonal (see [Voi17]). See [Sh] for more details.

Remark 5.5. Mingmin Shen explained to us that one can conversely deduce the integral Hodge conjecture on $F$ from that on $X$ (which holds by Voisin's result [Voi]). Indeed, in this case $P_{*}: C H_{1}(F) \rightarrow C H_{2}(X)$ is surjective ( [M, Theorem 3.1] and [Sh14, Theorem 1.1] show that $P_{*}$ is surjective modulo multiples of $h^{2}$ and a separate argument shows that $h^{2}$ is also in the image). From this we deduce that the image $\alpha(\gamma)$ of a class

$\gamma \in H^{3,3}(F, \mathbb{Z})$ is homologous to a cycle of the form $P_{*}(\Gamma)$, where $[\Gamma]=\gamma$. In particular $\gamma$ is algebraic.

\section{References}

[AV] E. Amerik, M. Verbitsky, Rational curves on hyperkähler manifolds, Int. Math. Res. Notices 23 (2015), 13009-13045. 
[Ap] A. Apostolov, Moduli spaces of polarized irreducible symplectic manifolds are not necessarily connected, Ann. Inst. Fourier vol. 64 (2014) no. 1, 189-202.

[BL] B. Bakker, C. Lehn, A global Torelli theorem for singular symplectic varieties, arXiv: 1612.07894.

[BCC $]$ E. Ballico, F. Catanese and C. Ciliberto, Trento examples, Classification of irregular varieties (Trento, 1990), Lecture Notes in Math., vol. 1515, Springer, Berlin, 1992, pp. 134-139.

[Be] A. Beauville, Variétés Kählériennes dont la première classe de Chern est nulle, J. of Diff. Geom. 18 (1983).

[BD] A. Beauville and R. Donagi, La variété des droites d'une hypersurface cubique de dimension 4, C.R.Acad.Sc. Paris 301, 703-706.

[BHT] A. Bayer, B. Hassett, Y. Tschinkel, Mori cones of holomorphic symplectic varieties of K3 type. Ann. Sci. Sci. de ENS. Vol. 48, No. 4 (2017) 941-950.

[BO] O. Benoist and J.C. Ottem, Failure of the integral Hodge conjecture for threefolds of Kodaira dimension zero. To appear in Commentarii Mathematici Helvetici (2018)

[BS] S. Bloch and V. Srinivas, Remarks on correspondences and algebraic cycles, Amer. J. of Math. 105 (1983) 1235-1253.

[CMSB] K. Cho, Y. Miyaoka, and N. I. Shepherd-Barron. Characterizations of projective spaces and applications. Higher dimensional birational geometry (2002): 1-88.

[CMP] F. Charles, G. Mongardi and G. Pacienza, Families of rational curves on holomorphic symplectic varieties and applications to 0-cycles, arXiv:1907.10970.

[CK] C. Ciliberto, A. L. Knutsen, On k-gonal loci in Severi varieties on general K3 surfaces, J. Math. Pures Appl. 101 (2014), 473-494.

[FKP] F. Flamini, A. L. Knutsen, G. Pacienza, Singular curves on a K3 surface and linear series on their normalizations, Internat. J. Math. 18 (2007), no. 6, 671-693.

[GHS] V. Gritsenko, K. Hulek, G. K. Sankaran, Moduli spaces of irreducible symplectic manifolds. Compos. Math. 146 (2010), no. 2, 404-434.

[HT] B. Hassett and Y. Tschinkel, Moving and ample cones of Holomorphic symplectic fourfolds, Geometric and functional analysis vol. 19 (2009) no. 4 1065-1080.

[KLM] A. L. Knutsen, M. Lelli-Chiesa, and G. Mongardi, Severi Varieties and BrillNoether theory of curves on abelian surfaces, J. Reine Angew. Math. 749 (2019), $161-200$. 
[KLM2] A. L. Knutsen, M. Lelli-Chiesa, and G. Mongardi, Wall divisors and algebraically coisotropic subvarieties of irreducible holomorphic symplectic manifolds, Trans. Amer. Math. Soc. 371 (2019), no. 2, 1403-1438.

[LP] Ch. Lehn and G. Pacienza, Deformations of singular symplectic varieties and termination of the log minimal model program, Alg. Geom. 3 (2016) no. 4, 392-406.

[Ma] E. Markman, A survey of Torelli and monodromy results for HyperKähler manifolds, Proc. of the conference "Complex and Differential Geometry", Springer Proceedings in Mathematics (2011), Volume 8, 257-322.

[Ma2] E. Markman, Lagrangian fibrations of holomorphic symplectic varieties of $K 3^{[n]}$-type, Algebraic and Complex geometry, 241-283, Springer Proc. Math. Stat. 71.

[Mat] D. Matsushita, On isotropic divisors on irreducible symplectic manifolds, arXiv: 1310.0896

[M] R. Mboro, Remarks on the $\mathrm{CH}_{2}$ of cubic hypersurfaces. arXiv preprint arXiv:1701.04488 (2017)

[Mo] G. Mongardi, A note on the Kähler and Mori cones of hyperkähler manifolds, Asian. J. Math. 19 (2015), 583-592.

[MP] G. Mongardi, G. Pacienza, Polarized parallel transport and uniruled divisors on deformations of generalized Kummer varieties, Int. Math. Res. Not. rnw346, https://doi.org/10.1093/imrn/rnw346 (2017).

[MP2] G. Mongardi, G. Pacienza, Density of Noether-Lefschetz loci of polarized irreducible holomorphic symplectic varieties and applications, arXiv:1804.09440.

[N] V.V. Nikulin, Integral symmetric bilinear forms and some of their applications, Math. USSR Izv. vol. 14 (1980), 103-167.

[OSY] G. Oberdieck, J. Shen, and Q. Yin. Rational curves in the Fano varieties of cubic 4-folds and Gromov-Witten invariants. arXiv preprint arXiv:1805.07001 (2018).

[OG] K. G. O'Grady, Higher dimensional analogues of K3 surfaces, Current developments in algebraic geometry, 257-293, Math. Sci. Res. Inst. Publ., 59, Cambridge Univ. Press, Cambridge, 2012.

[Ran] Z. Ran, Hodge theory and deformations of maps, Compositio Math. 97 (1995), 309-328.

[Sh] M. Shen. Rationality, universal generation and the integral Hodge conjecture. arXiv: 1602.07331.

[Sh14] M. Shen. Prym-Tjurin constructions on cubic hypersurfaces. Documenta Mathematica 19 (2014). 867-903. 
[SV16] M. Shen and C. Vial. The Fourier transform for certain hyperKähler fourfolds. Vol. 240. No. 1139. American Mathematical Society, 2016.

[Tot13] B. Totaro, On the integral Hodge and Tate conjectures over a number field, Forum Math. Sigma 1 (2013).

[Voi] C. Voisin, On integral Hodge classes on uniruled or Calabi-Yau threefolds, Moduli spaces and arithmetic geometry, Adv. Stud. Pure Math., vol. 45, Math. Soc. Japan, Tokyo, 2006, 43-73.

[Voi07] C. Voisin. Some aspects of the Hodge conjecture, Jpn. J. Math. 2 (2007), no. 2, 261-296.

[Voi14b] C. Voisin, Remarks and questions on coisotropic subvarieties and 0-cycles of hyper-Kähler varieties, "K3 Surfaces and Their Moduli", Progress in Math, Proceedings of the Schiermonnikoog conference 2014, Birkhäuser.

[Voi17] C. Voisin. On the universal $\mathrm{CH}_{0}$ group of cubic hypersurfaces J. Eur. Math. Soc. 19, (2017), 1619-1653.

[Wi1] B. Wieneck, Monodromy Invariants and Polarization Types of Generalized Kummer Fibrations, Math. Z. 290 (2018), no. 1-2, 347-378..

[Wi2] J. Wierzba, Contractions of symplectic varieties, J. Alg. Geom. vol. 12 (2003) $507-534$.

Department of Mathematics, Alma mater studiorum Università di Bologna, Piazza di Porta san Donato 5, 40126 Bologna, Italy

Email: giovanni.mongardi2@unibo.it

Department of Mathematics, University of Oslo, Box 1053, Blindern, 0316 Oslo, Norway Email: johnco@math.uio.no 